bodies two or three times as large as the usual spores were found (chlamydospores?).

Cultures on hanging-drop agar showed the mycelium to branch irregularly, the spores springing from the sides by slender stems or grouped around the ends of the branches as pictured in the illustration.

This case adds another one from the Missouri Valley, where, according to Reudiger, ${ }^{4}$ by far the largest number has been found.

Unfortunately the patient disappeared about the time the cultures were obtained and efforts to locate him were unsuccessful.

\section{CANCER OF THE RECTUM AND} THE PELVIC COLON

STATISTICAL AND CLINICAL REVIEW OF FOUR HUNDRED AND NINETY-ONE CASES *

JEROME M. LYNCH, M.D.

Assistant Surgeon, Medical Reserve Corps, U. S. Navy; Fellow of the American College of Surgeons NEW YORK

I have seen to date 491 cases, which my associate, Dr. Giles, has tabulated (Table 1). Fifty-one patients died and 253 recovered, a mortality of 16 per cent. for

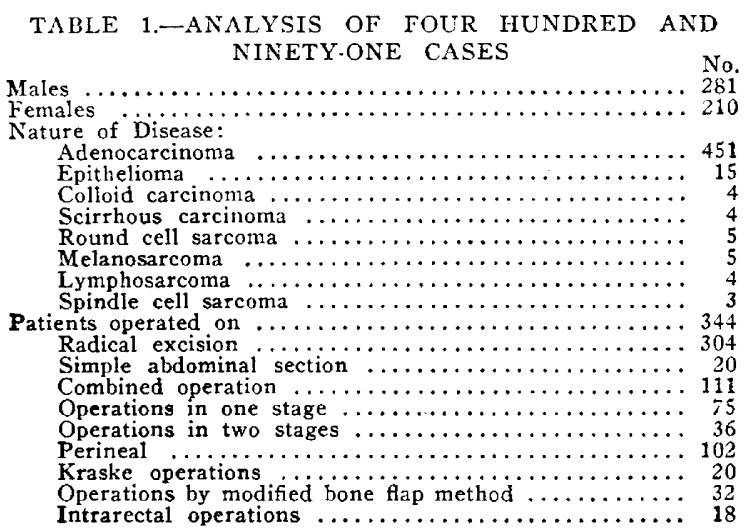

radical excision. The remaining forty-one cases not included in the series were colostomies and palliative operative procedures for inoperable cases.

I feel very strongly that early colostomy in inoperable cases is as vital to the patient as early operation in operable cases. And I further feel that it is a great injustice to put off this operation until the patient is moribund. If colostomy is indicated, it certainly should be performed before the individual has become so weakened by the disease that the operation will be of no benefit.

There are so many inherited misconceptions with regard to cancer, and they are so firmly rooted in the minds of the laity, that it will require a long time to dislodge them. Reeducation is essential if we wish to progress. Textbooks on surgery, except the more recent, lay stress on age, pain, cachexia, loss of weight, and tumor as the cardinal symptoms of cancer. This is very misleading. Cancer knows no age; 0.5 per cent. of these cases are in children under 9 years of age; 2.5 per cent. of the patients are under 19, and in

4. Reudiger, Gustav: Sporotrichosis in the United States, Jour. Infect. Dis. 1912, 11, 193-206.

Kead before the Section on Gastro.Enterology and Practology at the Sixty-Eighth Annual Session of the American Medical Association, New York, June, 1917. my personal experience 7 per cent. were under 30 and 9 per cent. were between 30 and 35 years of age.

The symptoms mentioned in the textbooks are those associated with the last or inoperable stage of cancer. Many of these patients seen by us had had unmistakable symptoms of the disease for over a year, although some of them had gained in weight.

The average duration of symptoms before admission is just over 8 months.

TABLE 2.-AGE OF CANCER PATIENTS

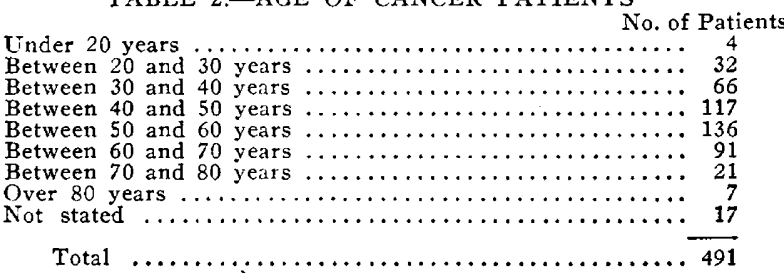

Cachexia seldom appears until cancer is far advanced, and then principally in old persons and in those with cardiovascular diseases. One does not often associate pain with cancer of the rectum, except as a terminal symptom, and when the tumor is situated adjacent to the anus. In rectal malignancy the tumor can usually be felt with the finger, and on this examination alone a diagnosis can be made; but when the disease is situated in the sigmoid or the colon, the diagnosis is made in the former by means of the proctoscope. In the latter case, I admit that many difficulties are encountered in arriving at a conclusion. However, the roentgen ray and the natural inhibition usually associated with malignancy, together with the presence of blood in the stool and the reaction of occult blood, afford material aid in differentiating benignancy from malignancy. Except in advanced cases, an abdominal cancer can very seldom be felt, and then only in patients with a relaxed abdominal wall. Frequently, when a mass is found, it is ais accumulation of fecal matter above the site of the growth, and not the tumor.

It is fortunate for the individual if this accumulation of fecal matter occurs early, as then, at operation, the malignancy is recognized. The late Dr. William Bull told me that he believed the day was not far distant when the public would be so educated as to accept exploratory laparotomy in every instance in which there is doubt. He lamented the difficulties he

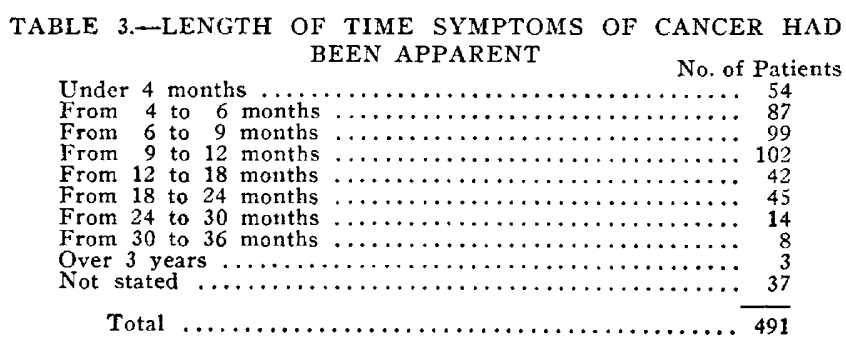

encountered in trying to convince patients that this was the proper course to pursue. If we could on!y remove from the minds of our patients preconceived notions of the immodesty of a rectal examination and insist on this in every case, many tumors would be discovered in their incipiency. Especially must we eliminate malignancy in cases in which there are cortstipation, diarrhea, hemorrhoids and loss of blood.

When it is realized that 10 per cent. of my patients had had an operation for hemorrhoids within a few 
months of the time when I first saw them and that they might have been spared the suffering which followed those operations, we must feel convinced of the necessity of being thorough, and conclude that hemorrhoids are all too often the symptom of a worse malady.

Twenty per cent. of these patients had an incorrect diagnosis and were treated for stomach and enteric disease, when, in reality, they were suffering from a malignancy remote from these organs. It is well known that esoteric stomach symptoms are very misleading, but here again we are confronted by the lack of thoroughness.

We should make it a rule, no matter in what branch of medicine or surgery we specialize, to see that every patient referred to us has the thorough examination to which he is entitled. One fails to be thorough if one overlooks anything, and is guilty of neglect toward that patient.

Dr. John B. Murphy's advice to me, when I started to practice, was to examine every patient thoroughly, and if he refused such an examination, to decline to treat him. Nicholas Senn, on so many occasions, reiterated the necessity of a digital examination that I am sure most of his students followed his advice.

Many cases of carcinoma of the rectum are turned down as inoperable without due consideration. This decision is often influenced by the high mortality in the hands of some surgeons. Also, because in the

TABLE 4.-RESECTIONS IN CANCER PATIENTS

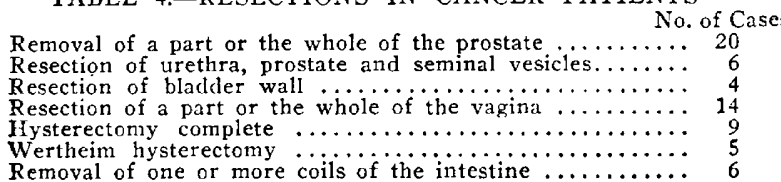

past, a large tumor mass with the involvement of some unimportant structure was considered as a contraindication to a radical operation. I recall several instances in which this decision influenced the surgeon. But we should remember that cancer here remains stationary for a very long time, that the patient is facing certain death unless some effort is made to eradicate the growth, and that even if the patient should die in the attempt to save him, he is at least spared a great deal of horrible suffering. On many occasions I have found it necessary to remove the uterus, resect the vagina, remove a portion or the whole of the prostate, resect the seminal vesicles, resect the urethra, perform a radical Wertheim operation, resect several coils of the intestine and resect the bladder wall.

One must expect a high mortality in such radical operations; but the percentage of cures is a sufficient justification to encourage us to continue and, if necessary, to do even more radical work. Dellman, in 1916, giving statistics for cancer in the Netherlands, says, in conclusion, that the more radical treatment of cancer nowadays is curing so many patients that the day is approaching, if not already here, when the number of deaths from malignant disease will no longer be an index of the actual prevalence of cancer. This is certainly encouraging, coming as it does from one who has evidently studied the subject very thoroughly.

The choice of operation in cancer of the rectum is always perplexing, and one that cannot be decided offhand; but it is safe to say that there are four operations, any one of which, when indicated, offers a solution to the problem.
Patients very often insist on knowing beforehand whether they will be left with a hole in their side, or whether it will be possible to restore the anus to its original site. Some men, in an effort to please, make contracts that they are unable to fulfil. This is unwise. In such a vital matter, the surgeon should insist that he be given full power to do what in his judgment is to the best interest of his patient. No surgeon should tie his hands beforehand and feel compelled, because of a previous understanding, to perform an incomplete operation when a slightly more radical procedure might prevent recurrence. I myself prefer to place the anus at its original site; but I never hesitate to leave a patient with a colostomy if by so doing I can get a better result. I never consider placing the anus high up in the sacrum, or in any other situation than the original site, because I believe that a colostomy is preferable to a stoma in any other than the normal position.

I agree with the surgeons who state that perfect continence can never be attained by attempts at restoration of the intestine to the normal anal site. Yet I have so many cases in which patients have enjoyed great comfort with this imperfect continence that $\mathrm{I}$ am convinced that this operation has a place in surgery.

Everything being relative, this applies with equal force to incontinence; and I feel confident that if a census could be taken of patients with relative continence, after a radical operation of the rectum, none of them would be willing to have a colostomy, in preference to a relative continence, with the anus in its normal situation.

My operation of choice is the combined operation, with the anus in its normal site in the perineum. This gives one the advantage of being able thoroughly to explore the abdomen. I know from experience that the under and diaphragmatic surfaces of the liver are first involved, and that only by abdominal exploration can this be determined. There are other advantages, namely, that one can perform a practically bloodless operation, and remove all the interglandular tissue and fat with a minimum of traumatism.

It is my custom, whenever possible, to block the sympathetic by the combined extradural and hypogastric anesthetization, as I feel that, by this method, shock, which is a bugbear to all surgeons in this operation, is obviated. I suggested to Dr. Halstead that this procedure accounted for my better. results since I have followed it; but he seemed to think that it was due rather to a more perfect technic than to the combined blocking. Still, I nevertheless feel a good deal like the surgeons of the old school after they had washed their hands in mercuric chlorid, that it is at least a step in the right direction, adds no risk to the operation, and is in keeping with modern thought. The work of Crile confirms me in my belief, and so long as it does not add to the danger or the length of the operation, it seems well worth while.

The fact that a perineal operation was performed by me completely under sacral anesthesia must convince the most pessimistic of its value as a distinct method in blocking sensory impulses.

Whether the combined operation should be performed in one or two stages can only be decided at operation, and the surgeon is guided by his own experience and judgment. Often the anesthetist becomes alarmed during the course of the operation, but the 
surgeon familiar with this operation can always see the danger signal ahead. I prefer to perform the operation in one stage whenever feasible, but I am not inflexible in my judgment and never hesitate, if I feel that there is extra risk, to divide it into two stages.

The perineal operation is indicated in very fat persons and in those so debilitated that any form of abdominal exploration would be almost sure to result in death. In looking over my perineal operations, especially in that decade when this was the operation of choice, I find so many good results that it is hard to believe that this method should have such a limited field of usefulness.

Kocher many years ago pointed out that extensive glandular enlargement does not necessarily mean that those glands themselves are carcinomatous. I have had those glands examined at levels, and at various distances, from the growth, and in the majority of cases they have proved to be merely inflammatory. However, at the present date, it is much safer to explore the abdomen than to confine oneself to the perineal operation alone.

In all cases, no matter what the operation, I remove the coccyx as a matter of routine. It affords far greater room, obviates the pain that follows the operation when the coccyx is not removed, permits of thorough drainage, and adds materially to the comfort of the patient in subsequent dressings.

When the growth is situated near the anus, it seems advisable to perform a colostomy first. At the end of ten days, a wide incision is made of the tuberosities on both sides, and the growth is removed en bloc after the ischiorectal fossae are thoroughly cleaned out. I have succeeded in restoring the anus to its original site, and I have obtained a relative degree of continence for so extensive an operation.

$I$ have found it to be of advantage to pack the pelvis by means of a device that has given great satisfaction from the standpoint of drainage, and has spared the patients unnecessary suffering during the removal of the gauze. No doubt this is nothing new, and has probably been used with equal satisfaction by others; but I mention it for the benefit of those who are unfamiliar with its advantages. A rubber glove, from which the upper halves of the fingers have been cut, and in which a small opening has been made in the palmar and dorsal surfaces to permit of drainage, is passed into the pelvis as tightly as necessary to control oozing and to fill up the dead spaces posterior to the bowel. At the end of forty-eight hours the gauze can be removed, the wound irrigated, if necessary, or desirable, and the glove repacked.

I have long since abandoned resection of the restum, as early experience has taught that stricture inevitably followed. This is owing to the fact that the rectum has a terminal blood supply, and not, as is commonly supposed, to the lack of peritoneal covering. It is an axiom in pathology that the amount of connective tissue is inversely proportionate to the blood supply.

Kraske's operation has been abandoned by most surgeons, and I see very little indication for it in cancer of the rectum.

The sigmoid and cecum are two of the most variable organs of the body. The sigmoid, particularly, varies within wide limits, as in the case of Hirschsprung's disease. It possesses few lymphatics, ,has a dense fascial covering, and, for this reason, sigmoidal growths remain stationary for a long time. On account of its wide variation, it lends itself easily to resection.

There are many drawbacks to end-to-end anastomosis in this region, on account of the blood supply and the danger of leakage. Recognizing this, Mikulicz, Tuttle and others have suggested a three stage operation which has a low mortality, but like all safe procedures, has many inconveniences, in that the patient is incapacitated for a long time, and it may require more than one operation to close the stoma. It would also seem that the danger of metastasis would be enhanced by the subsequent handling and pressure, and by allowing the growth to remain in situ many days after the first operation.

For several years, except in cases of obstruction and in patients that are greatly debilitated, I have used the following technic: The sigmoid naturally rests in the iliac fossa, and this can be taken advantage of to make a lateral anastomosis between the two limbs of the sigmoid. The mesentery should be tied as in the Mikulicz operation. Both legs of the sigmoid are then brought together by a peritoneal suture on the inside. The intestine is mobilized toward the center, the pelvic and small intestines being carefully walled off. Following this, an anastomosis is made on the outer side of the parallel limbs. After the anastomosis has been completed, the sigmoid is allowed to fall toward the iliac fossa and is sutured to the parietal peritoneum, dorsad, cephalad and caudad. A small cigaret drain is placed above and below the anastomosis, and the abdominal wound is closed, except for the skin. Both limbs are double clamped, and the growth is removed with the cautery. After this the ends of the bowel are inverted, and the skin is closed in the usual manner. If leakage should occur it will be practically shut off by the two walls of the peritoneun?. If there is any trouble with the anastomosis, the skin is opened up, and the cephalad portion of the bowel is opened and clrained.

108 East Sixty-Fifth Street.

\section{ABSTRACT OF DISCUSSION}

Dr. George B. Evans, Dayton, Ohio: I have never seen a patient with carcinoma under 55 years of age. In cases in which the pelvis is much involved-by that I mean the vagina, uterus, ovaries and tubes, seminal vesicles, prostate and posterior urethra-I believe we should not extirpate or resect. Although we may give our patients temporary relief, their secondary sufferings are greater. I have never used the cautery because $I$ feel it is better for me to have my finger up in the wound.

As Dr. Lynch has said, the question of diagnosis is by far the most important in these cases, and we should make it with the aid of the proctoscope, sigmoidoscope and roentgenogram. We should never operate in any case of carcinoma until we have given the patient's kidneys a thorough consideration. We should never operate on these patients until we have brought the phenolsulphonephthalein test to our aid. In the first ten years of my work, nearly all of my cases were operable, as I thought in those days. In the last ten years I do not think that in all the cases I have seen 10 per cent. were operable. I find that all the organs in these cases are involved. Therefore, and in conclusion I wish to emphasize that $I$ believe we are going back to where we were, so far as the good of the patient is concerned-back to colotomy.

Dr. Frank C. Yeomans, New York: Every one in speaking of cancer of the rectum and cancer of the colon emphasizes early diagnosis, saying that if the surgeon gets the patient early enough he will cure him, no matter where the 
cancer is. Then they urge that we examine the patients carefully. I think all this is very good precept and practice; but many men who have been practicing for years never examine patients carefully. They do not take postgraduate medical courses, and they were not taught in the undergraduate schools the method of making a routine examination. The only way that we can hope to succeed in this, I believe, is to have courses of instruction in enteroproctology introduced into the undergraduate medical schools and make this a compulsory part of the curriculum, so that a student may become familiar with the average pathology of the bowel and know how to use the ordinary instruments of precision. Then, if he cannot interpret the findings, he will, at least, have the wisdom to confer with some one competent to make the diagnosis before the disease has progressed to a stage where it is beyond relief. Until that time the entire profession will not receive adequate instruction, cancer of the bowel will not be recognized early, nor will the patient receive the benefit of early operation.

Dr. William M. Beach, Pittsburgh: I should like to ask Dr. Lynch the tenure of life following these cases.

Dr. Dwight H. Murray, Syracuse, N. Y.: The microscope does not tell us the histologic factor. This may seem strange. I will explain by saying that in the examination of the cancer tissue removed, the pathologist will say that all of the growth has been removed and that the microscope does not show any cancerous tissue on the outside of the growth. In my opinion the microscope cannot show tissue which has been invaded and which has not yet shown pathologic changes that the microscope will reveal. I saw this thoroughly demonstrated in a case in which operation was performed a year ago.

Dr. John L. Jelks, Memphis, Tenn.: I am so pessimistic with regard to cancer of any part of the alimentary tract that I feel almost inclined to offer a resolution that it would be the sense of this assembly that late radical operations for cancer of the stomach and intestines are hardly warranted. I have operated and operated for cancers of the stomach, colon and rectum and the patients are all dead, or, if not already dead, going to die a little bit later of cancer of the liver. What is the use? I think it is best to turn the patients down. A number of men have said much could be done if we have "early cases." In the "early cases" the patients will not dream of having, an operation and in the late cases we should not dream of operating.

Dr. Lovis J. Hirschman, Detroit: I hope this paper will go out as a message from the enteroproctologist to the general surgeon and everybody doing surgery, to mark the passage of mutilating operations for the excision of a cancer; that the day of the sacral anus is over. I think the time has arrived when we must tell the profession at large that if they cannot do anything else than colostomize and implant the anus anywhere but at its normal site, they had better do nothing.

Dr. Jerome M. Lynch, New York: I am really sorry that my friend Dr. Jelks feels as he does; it is very unfortunate, for Dr. Jelks has many friends and a large following and the result will be that a great many patients with cancer who should be operated on will not be operated on. The statistics of Mayo and other men show that a great deal has been done in cancer.

Dr. Beach asked me for the tenure of life in these cases. Twenty-six per cent. of the patients are alive after three years (all these statistics will be published); two or three over sixteen years. When the average patient comes for operation he is over 50, and if he lives sixteen or seventeen years, he is going about the limit. So far as I can see, the results are very encouraging, and notwithstanding the pessimistic view taken by some surgeons, I shall still continue to perform these operations, and it will just mean holding the same position now that Samuel Sharpe held in 1820. Then he said, "Some men are so pessimistic about operation for cancer that they would rather let the patients die. In my own personal experience I have saved so many patients that I shali still continue to operate." It is almost 100 years since that time and we have the same obstructive pessimism to contend with.

\section{MIXED INFECTION WITH THE PNEU- MOCOCCUS IN EPIDEMIC MENINGITIS *

\author{
GEORGE MATHERS, M.D. \\ CHICAGO
}

In a recent communication, Netter and Salanier ${ }^{2}$ report twenty-two instances of epidemic meningitis in which pneumococci as well as meningococci were found in the spinal fluid. These cases of mixed infection exhibited all the symptoms of meningococcic meningitis, but did not respond to serum treatment and terminated fatally. It is my purpose to call attention to the importance of these observations, and to report an example of this type of meningeal infection.

W. D., a boy, aged 7 months, entered the Cook County Hospital, Sept. 28, 1917, with all the physical signs of meningitis. According to the mother, the illness began suddenly two days before with convulsions, vomiting, stiffness and retraction of the neck, and a staring expression of the eyes. Otherwise the history was negative. The noteworthy physical findings were blindness, marked opisthotonos, orthotonus, and a positive Kernig's sign. The temperature was $103 \mathrm{~F}$. and the pulse rapid and weak.

The spinal fluid was under increased pressure, opalescent, and contained 1,000 cells per cubic millimeter, 98 per cent. of which were of the polymorphonuclear type. Gram-positive and gram-negative intracellular and extracellular diplococci were present in the stained smears. The urine contained a trace of albumin and an occasional leukocyte, and the Wassermann test was negative. During the twenty days in the hospital, 185 c.c. of antimeningococcus serum were given intraspinally with no results. The temperature remained high and the child gradually became weaker and died, Oct. 18, 1917. A necropsy was not made.

During the course of the disease two bacteriologic examinations of the spinal fluid were made. In each instance a gram-negative and a gram-positive diplococcus were found in the cultures. The gram-negative organism had all the morphologic and cultural characteristics of the meningococcus, and was agglutinated by a polyvalent antimeningococcic serum in a dilution of $1: 800$. The gram-positive diplococcus was found to be a typical pneumococcus of Group I type. In the plate cultures of the spinal fluid in both instances, the meningococcus colonies were more numerous than the pneumococcus colonies. The spinal fluid had a faint greenish tint, and at no time was markedly purulent. Precipitin tests of the spinal fluid, in which the specific serums for both organisms were used, yielded megative results.

These observations emphasize the importance of frequent cultural examinations of the spinal fluid in all cases of epidemic meningitis, especially those in which antimeningococcus serum does not prove efficacious. Furthermore, they indicate that mixed infection may occur in meningococcic meningitis as in other infectious diseases, and exert an unfavorable influence on the clinical course. In the instance of mixed pneumococcus and meningococcus infection, combined serum treatment, as advocated by Netter, would be indicated. In regard to the general significance of this new observation in epidemic meningitis, it is sufficient to say that the importance of mixed infection in acute infectious diseases cannot be overestimated. The presence of two virulent organisms in the same pathologic lesion must not be neglected on the basis that one is a secondary invader; the activities of each organism must be reckoned with in the treatment.

\footnotetext{
* From the Memorial Institute for Infectious Diseases.

1. Netter and Salanier: Bull. et mém. Soc. méd. d. hôp. de Paris,
$1917,41,289$.
} 